\title{
ZnO Nanostructured Thin Films via Supersonic Plasma Jet Deposition
}

\author{
Chiara Carra ${ }^{1}$, Elisa Dell'Orto ${ }^{1}$, Vittorio Morandi ${ }^{2}$ (I) and Claudia Riccardi ${ }^{1, *(1)}$ \\ 1 Dipartimento di Fisica "Giuseppe Occhialini", Università degli Studi di Milano-Bicocca, Piazza della Scienza 3, \\ I-20126 Milan, Italy; c.carra@campus.unimib.it (C.C.); elisa.dellorto@unimib.it (E.D.) \\ 2 Institute for Microelectronics and Microsystems, Italian National Research Council, Bologna Unit, \\ Via Gobetti 101, I-40129 Bologna, Italy; morandi@bo.imm.cnr.it \\ * Correspondence: claudia.riccardi@unimib.it; Tel.: +39-0264482314
}

Received: 15 July 2020; Accepted: 8 August 2020; Published: 13 August 2020

\begin{abstract}
Zinc Oxide nanostructured thin films were grown by a novel plasma assisted vapour deposition method, which aims to combine the versatility of deposition processes that are mediated by plasma with the capability to control particles diffusion and nucleation. For this purpose, the proposed approach spatially separates into two different vacuum chambers the creation of zinc oxide from a metalorganic precursor from the actual film growth, thanks to the extraction of a supersonic jet of plasma seeded by the precursor fragments. The characterization of the reactor in different plasma conditions has been carried out by means of optical emission spectroscopy (OES). ZnO films with different degrees of purity, thickness uniformity, as well as different morphologies can be obtained varying the deposition parameters. The samples profiles have been collected in order to evaluate deposition rates and films uniformity. The as-prepared as well as annealed thin films were characterized by attenuated total reflection Fourier transform infrared spectroscopy (ATR-FTIR) to evaluate their chemical composition and purity. According to Raman analyses, the annealed samples are high-purity wurtzite-type crystalline zinc oxide films. Atomic force microscopy (AFM) and scanning electron microscopy (SEM) confirm a surface morphology characterized by columnar structures.
\end{abstract}

Keywords: nanostructured thin films; zinc acetylacetonate; $\mathrm{ZnO}$; plasma deposition and supersonic plasma jet

\section{Introduction}

Nanotechnology proves to be extremely attractive for successful applications in various fields, such as electronics, photovoltaics, environmental protection, and chemical industry. In particular, thin-films science and technology play a crucial role in the development of these modern and advanced technologies [1,2]. Depending on the nanostructures features, thin films show very different electrical and optical properties from their bulk crystalline counterparts. The downscaling process in thin film fabrication has two major effects; firstly, the exposed surface appears to be much greater than the thickness of the structures, thus allowing for a better efficiency in surface processes and chemical reactivity; secondly, the dimensional quantum confinement alters the band structures of the material leading to new and exotic optical and electrical characteristics [3]. To maximize the advantages of this technology, it is possible to study and develop techniques to deposit nanostructured thin films of desired characteristics by acting directly on the growth processes controlling the clusterization. The zinc oxide, object of this work, is a semiconductor having a direct band gap of 3.10-3.37 eV at room temperature [4]. High-quality $\mathrm{ZnO}$ bulk crystals have a large exciton binding energy of $\sim 60 \mathrm{meV}$ and a effective total radiative and nonradiative lifetime of excitons of the order of 
$1 \mathrm{~ns}$ [5]. As a result, $\mathrm{ZnO}$ posses an intense near-band-edge excitonic emission at room or higher temperatures and it holds great interest as active material. Additionally, $\mathrm{ZnO}$ has a high-energy radiation stability and amenability to wet chemical etching, making it a suitable candidate for space application and fabrication of microelectronics devices, respectively. Recently, zinc oxide nanostructures with controlled dimension, size, porosity, crystal facets, and mesoscale architectures have raised great interest in various applications in sensoring material, photocatalysts, solar cells, and light-emitting applications [6-9]. Among other growth methods, such as sol-gel processes, molecular-beam epitaxy, and RF magnetron sputtering, the plasma-assisted chemical vapor deposition is particularly interesting because of its high growth rate and large-scale applications. The high vacuum conditions guarantee a lower surface damage and the low temperature better adhesion and smaller grain size. Moreover, the Plasma Assisted Supersonic Jet Deposition has proven to be successful for the growth of high-quality $\mathrm{ZnO}$ films with interesting nanostructured morphology.

\section{Materials and Methods}

An innovative method for deposition of zinc oxide nanostructured thin films is the Plasma Assisted Supersonic Jet Deposition (PA-SJD). The PA-SJD is a patent designed and developed at the Centro PlasmaPrometeo of the University of Milano-Bicocca [10]. This method aims to combine the versatility of the deposition processes that are mediated by plasma with systems of controlled aggregation of clusters, separating into two distinct phases the process of the creation of zinc oxide and the growth of thin films on the substrate. Changing the process parameters, different degrees of purity, and uniformity of the films can be obtained, as well as different morphology. The reactor is constituted by two vacuum chambers, in order to decouple the deposition procedure. In the first chamber, a very reactive and dense plasma is generated by a radio frequency inductively coupled source. In this highly reactive environment, a metal-organic precursor in gaseous form is injected and then dissociated by the plasma to obtain zinc oxide nanoparticles. The second chamber is kept at a lower pressure and it is connected to the first one through a nozzle, thus a supersonic jet of plasma is generated. The jet contains the particle seeds to be deposited, which impact on the substrate starting the nucleation processes. Consequently, the reactions that take place in the plasma phase are decoupled from aggregation phenomena that occur during the supersonic expansion, effectively controlling the growth of a nanostructured thin film. In this experimental plasma conditions, the supersonic jet allows for the deposition of clusters on the substrate favoring the growth of columnar structures. The low collisionality of particles in the jet prevents reassembling processes causing the creation of nanostructures of very small size [11]. In order to characterize the deposition system each step is taken into account; firstly, a thermal analysis on the precursor has been performed to estimate its injection efficiency, consisting of vapor pressure curves measurement, thermogravimetrical analysis (TGA), and differential scanning calorimetry (DSC). During the deposition process, plasma is monitored through optical emission spectroscopy (OES), in order to evaluate precursor flux and its dissociation. Thin films are deposited under different conditions and characterized by means of profilometry, Attenuated Total Reflection-Fourier Transform Infrared spectroscopy (ATR-FTIR) spectroscopy, Raman spectroscopy, Atomic Force Microscopy (AFM), and Scanning Electron Microscopy (SEM). Determining purity, morphology, and structure proprieties provides the ability to tune the working conditions to obtain the desired thin film structures.

\subsection{PA-SJD Device}

A high level of control on the thin films growth can be achieved decoupling the processes of the precursor oxidation and the particles acceleration and deposition onto the substrate. This can be achieved by spatially separating the region where the precursor fragmentation in plasma phase occurs and the region where the deposition of clusters on a substrate takes place. The precursor is dissociated by a reactive plasma in the first chamber, by means of the converging nozzle a supersonic jet of gas and particles is extracted into the second chamber. During deposition processes, the substrates are placed 
near the Mach disk of the shock wave. Films characteristics, such as thickness, uniformity, purity, and morphology, can be tuned by changing plasma and jet parameters.

The main vacuum vessel, a stainless-steel cylinder ( $410 \mathrm{~mm}$ height, $160 \mathrm{~mm}$ inner radius), has been converted into two chambers, fabricating a cylindrical plasma chamber inside $(95 \mathrm{~mm}$ height, $62.5 \mathrm{~mm}$ inner radius) connected to the upper lid of the vessel. The experimental set-up is shown in Figure 1. The chambers are connected through a circular converging nozzle, having a diameter of $6.9 \mathrm{~mm}$, and it accelerates the gas to a sonic speed. The substrates are fixed on an aluminum movable holder placed at the desired distance from the nozzle, along the axis of the jet. A turbomolecular pump and a rotary pump provide the high vacuum conditions in the chambers (the limit pressure inside the deposition chamber is near $10^{-5} \mathrm{~Pa}$ ). The gate valve of the main pumping group can be partially closed, reducing the effective pumping speed, and allowing to set a desired pressure inside the deposition chamber $\left(\mathrm{P}_{\mathrm{d}}\right)$ with negligible variations to the plasma chamber pressure $\left(\mathrm{P}_{\mathrm{p}}\right)$. The pressure ratio between the two chambers is defined as $R=P_{p} / P_{d}$ and it can be varied from 40 to 1 to model the supersonic jet geometry and energy. During the measurements, pressure in the chambers is monitored using two capacitance pressure gauges and a full range gauge, with an adequate pressure reading precision in the pressure ranges considered (1-10 Pa in the plasma chamber and 0.01-10 Pa in the deposition chamber). The metalorganic precursor is injected in the plasma chamber upon reaching a sufficiently high level power of the plasma discharge. The solid precursor, held in an aluminum tank outside the main vacuum vessel, is heated through a copper heating wire wrapped around the tank, powered by a variable autotransformer. The precursor temperature can be monitored by means of a thermocouple and varied to obtain different flows. A copper pipe, whose opening is controlled through a Swagelok on-off valve, allows the sublimed precursor inlet. The dissociation of the precursor occurs in a plasma of argon and oxygen: the first provides a stable plasma discharge and the second favors the precursor oxidation. Gases are injected directly in the plasma chamber through two different micrometric valves, in order to independently regulate their flows. The flow regimes in the plasma chamber at pressure of 4-8 Pa can be considered continuous, since the Knudsen number is $k_{n}<0.01$. Diffusion acts efficiently and the different species are well-mixed in the plasma. While inside the zone of silence in the deposition chamber, $k_{n}$ is calculated from the estimated gas mean free path, the collisional frequency of the supersonic jet, and the length of the Mach disk at the chosen distance from the orifice. The pressure range for the calculation was observed experimentally and it is between $0.13-0.25 \mathrm{~Pa}$. The estimated Knudsen number is $0.04<k_{n}<0.08$, therefore the flow regime in the supersonic jet region is transitional, guaranteeing an uniform jet over the substrates. The inductively coupled source is a two- and three-quarter loop planar antenna, made of copper wire and cooled by deionized water [12]. The antenna is placed inside the lower lid of the plasma chamber, shielded by a Teflon scaffold and covered by an alumina disk to reduce sputtering. Feeding the antenna through a $13.56 \mathrm{MHz}$ radiofrequency power generator (PFG $1600 \mathrm{RF}$, Huttinger Elektronic, Ditzingen, Germany) at $450 \mathrm{~W}$, an uniform and stable plasma discharge generates inside the plasma chamber. The supply RF signal is connected to the external edge of the coil with an L-type matching box along the transmission line and the internal end is connected to the ground. Inductive sources generate quite large plasma densities and relatively small electron temperatures at moderate pressures, in the range of 1-100 Pa. This favors dissociation of external, weakly bound chemical groups in organic precursors, partially preserving the backbone structures [13]. 


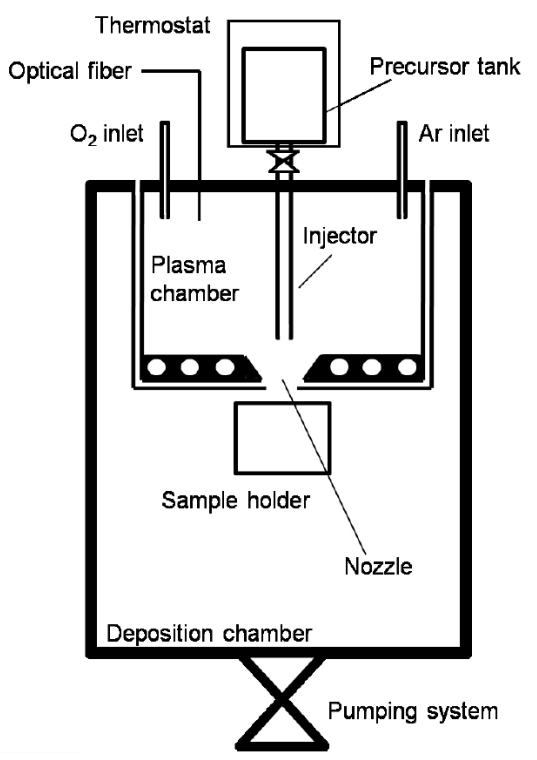

Figure 1. Schematic representation of the Plasma Assisted Supersonic Jet Deposition (PA-SJD) setup. The plasma chamber and the deposition chamber are connected by the converging nozzle.

\subsection{Diagnostics}

Thermogravimetric and differential scanning calorimetry analyses of precursor were conducted through a TGA/DSC1 STARe system (Mettler Toledo, Columbus, OH, USA), provided by a $10^{-6} \mathrm{~g}$ resolution balance. The analyses were conducted at the same time by warming the precursor aluminum tank with heating rate of $5{ }^{\circ} \mathrm{C} / \mathrm{min}$ at atmospheric pressure. The experiment was conducted in a nitrogen atmosphere in order to prevent unwanted oxidation reactions. The discharge emission spectra were recorded by means of an optical spectrometer (S2000, Ocean Insight, Largo, FL, USA), equipped with a $10 \mu \mathrm{m}$ slit and a 2048 pixels CCD, having a spectral band extending from $180 \mathrm{~nm}$ to $850 \mathrm{~nm}$ and a resolution of $0.3 \mathrm{~nm}$. Emission spectra were captured directly imaging the plasma region through a quartz view-port on the top flange with an IR enhanced optical fiber (FC-IR050-2, Avantes, Apeldoorn, The Netherlands). In order to obtain the real relative intensities of emission lines at different wavelengths, the system was calibrated with both a deuterium and halogen lamp (Avalight-DHc, Avantes, Apeldoorn, The Netherlands) to correct for the device sensitivity. The exposure time was optimized to match the CCD sensitivity, avoiding overcounts. Each spectra was averaged over multiple acquisitions for noise reduction and a dark spectrum with comparable statistics was subtracted. For what concerns thin films, the thickness analyzes were carried out through a Dektak 8 Stylus Profilometer (Veeco, Plainview, NY, USA), equipped with a diamond stylus and capable to provide a vertical resolution of $1 \AA$. The applied stylus tracking force was $15 \mathrm{mg}$. The profilometer could measure small vertical features ranging in height from $10 \mathrm{~nm}$ to $1 \mathrm{~mm}$. Surface chemical composition of samples was investigated by means of a Fourier Transform Infrared (FTIR) spectrometer (Nicolet iS10, Thermo Fisher Scientific, Waltham, MA, USA), equipped with a ATR sampling accessory (Smart iTR, Thermo Fisher Scientific, Waltham, MA, USA). For each spectrum, 64 coadditions scans with a spectral resolution of $2 \mathrm{~cm}^{-1}$ were recorded. Confocal micro-Raman scattering were performed using a LabRAM (Jobin Yvon, HORIBA, Kyoto, Kyoto, Japan) spectrometer and a He-Ne laser at $632.8 \mathrm{~nm}$ as excitation source, focusing the beam on a circular spot of $1 \mu \mathrm{m}$ in diameter through the optics of a microscope (Olympus, Shinjuku City, Tokyo, Japan) and an objective with magnification 100× and Numeric Aperture 0.90. The scattered light was detected by means of a Peltier- cooled silicon charge-coupled device cooled at $200 \mathrm{~K}$, after a notch filter (Kaiser Optical Systems, Ann Arbor, MI, USA) with $15 \mathrm{~nm}$ of bandwidth and a monochromator with a spectral resolution of $1 \mathrm{~cm}^{-1}$. Atomic force microscopy (AFM) measurements were carried out using a Solver P47-PRO (NT-MDT, Moscow, Russia), in semi-contact (tapping) mode on dry samples using HA-NC (High Accuracy Non Contact) silicon 
tips (NT-MDT, Moscow, Russia) of typical spring constant $3.5 \mathrm{~N} / \mathrm{m}$. The Scanning Electron Microscope (SEM) was a ZEISS 1530 (Jena, Germany) instrument equipped with a Schottky emitter and operated at a primary beam energy of $10 \mathrm{keV}$.

\section{Results and Discussion}

\subsection{Thermal Analysis of the Precursor}

A well suited precursor for PA-SDJ is a volatile organometallic compound, easily dissociated in an $\mathrm{Ar}-\mathrm{O}_{2}$ plasma. It should be non-toxic for health, environment and mechanical deposition system and it must have an accessible evaporation temperature. The precursor selected for the purpose of this work is zinc acetylacetonate. Zinc acetylacetonate hydrate (also referred as $\left.\mathrm{Zn}(\mathrm{acac})_{2}\right)$, having the chemical structure $\mathrm{Zn}\left(\mathrm{C}_{5} \mathrm{H}_{7} \mathrm{O}_{2}\right)_{2} \cdot x \mathrm{H}_{2} \mathrm{O}$ (with $0.7<x<1.3$ ), is a convenient zinc oxide precursor and an alternative for the mainly used zinc precursors, diethylzinc and dimethylzinc. Although it is preferred, since it is easier to handle, it has the inconvenient of being solid at room temperature with a sublimation temperature of the order of $100{ }^{\circ} \mathrm{C}$ [14].

The molecule of zinc acetylacetonate hydrate consists of formal single and double bonds, but, due to electronic delocalisation, the bonds between $\mathrm{Zn}, \mathrm{C}$, and $\mathrm{O}$ atoms are approximately equal. The relative strengths of the interatomic bonds are listed in Table 1. Zinc acetylacetonate is easily dissociated in plasma, mainly due to the oxidation reactions and dissociation by reactive species. Considering that the plasma temperature for the configuration utilised is approximately $1.5 \mathrm{eV}$, electrons can easily break the weakest $\mathrm{Zn}-\mathrm{O}$ bonds following the reaction:

$$
\mathrm{Zn}\left(\mathrm{C}_{5} \mathrm{H}_{7} \mathrm{O}_{2}\right)_{2} \longrightarrow \mathrm{Zn}^{2+}+2\left(\mathrm{C}_{5} \mathrm{H}_{7} \mathrm{O}_{2}\right)^{-}
$$

Table 1. Summary of the bond energies between carbon, oxygen, hydrogen, and zinc in zinc acetylacetonate hydrate [15].

\begin{tabular}{cccccccc}
\hline \multicolumn{7}{c}{ Bond Energies } \\
\hline Bond & $\mathrm{C}-\mathrm{C}$ & $\mathrm{C}-\mathrm{O}$ & $\mathrm{C}-\mathrm{H}$ & $\mathrm{Zn}-\mathrm{O}$ & $\mathrm{O}-\mathrm{H}$ & $\mathrm{C}=\mathrm{C}$ & $\mathrm{C}=\mathrm{O}$ \\
$\mathrm{eV}$ & 3.576 & 3.7 & 4.26 & 2.861 & 4.768 & 6.239 & 8.271 \\
\hline
\end{tabular}

Oxygen radicals are produced in the plasma because of the dissociation of oxygen molecules:

$$
\mathrm{O}_{2}+e^{-} \longrightarrow 2 \mathrm{O}+e^{-}
$$

The final component of the neutral chemistry that needs to be considered is the oxidation of the atomic zinc released by the decomposition of zinc acetylacetonate. However, thermal decomposition of $\mathrm{Zn}(\mathrm{acac})_{2}$ may be safely ignored, as no deposition is observed if plasma is not ignited [16]. This only leaves $\mathrm{Zn}$ as a potential deposition precursor. Thus, the deposition process can be simply described as a two-step process consisting of atomic zinc adsorption followed by oxidation:

$$
\begin{gathered}
\mathrm{Zn}(g)+\text { site } \longrightarrow \mathrm{Zn}_{(a d)} \\
\mathrm{Zn}_{(a d)}+\mathrm{O}_{(g)} \longrightarrow \mathrm{ZnO} \mathrm{O}_{(b u l k)}+\text { site }
\end{gathered}
$$

Although other species could participate in the oxidation step, atomic oxygen is greatly abundant under plasma operation and has the highest reactivity. To evaluate the temperature at which precursor had to be heated to guarantee a sufficient flux in the plasma chamber, it was necessary to preliminary measure the vapor pressure curves of the zinc acetylacetonate, as there were no previous reported measures in literature. The system to measure the precursor vapor pressure curves is a reservoir which contains the substance, maintained under vacuum by a rotary pump and provided with two inlets, one for the thermocouple and another for the pressure gauge. The tank was heated, in order 
to reproduce a thermostat, by means of a heat resistant wire wrapped around it, carrying a current generated by a variable autotransformer. The temperature was evaluated through the thermocouple, while the pressure in the container was measured by a capacitive pressure sensor. The system was connected to the pumping units through a valve. The sample was degassed before proceeding with measures by heating, cooling and, consequently, pumping of the residual gases. It should be emphasized that the measurement of vapor pressure curves appears very difficult at less than $1 \mathrm{kPa}$, as the data are often influenced by important systematic errors due to the sensitivity of the vacuum gauges and the degassing of the tank walls. Additionally, literature lacks in vapor pressures data and, where present, they can show differences of a few tens of percent. The measurements were conducted by heating the sample and by measuring the corresponding pressure inside the reservoir. Data exhibited a certain repeatability during the different sessions. Vapor pressure curves obtained are fitted with the Clausius-Clapeyron equation, in order to estimate the sublimation enthalpy of the compound. Clausius-Clapeyron equation can be rewritten arrested at the first order in a way more useful for the purposes of this work:

$$
P=\cos t+e^{a+\frac{b}{T}}
$$

where $P$ is the pressure of the gas, $T$ is its temperature, $a$ is a parameter depending on vapor nature, and $b$ is defined as $-\mathrm{E}_{0} / \mathrm{R}$, being $\mathrm{E}_{0}$ the sublimation enthalpy and $\mathrm{R}$ the gas constant [17]. In Figure 2 is shown the fit of a typical vapor pressure curve of $\mathrm{Zn}(\mathrm{acac})_{2}$ hydrate, obtained heating the tank at $2{ }^{\circ} \mathrm{C} / \mathrm{min}$. While Figure 3 shows the curve after the dehydration of the compound, carried out by pumping the gaseous content of the tank (presumably only due to water vapor) after heating at $60^{\circ} \mathrm{C}$ in vacuum conditions. Therefore curve is attributable to the anhydrous form of zinc acetylacetonate, and it is realized by increasing the temperature with a heating rate of $2{ }^{\circ} \mathrm{C} / \mathrm{min}$. From parameter $b$ of Equation (1) is therefore calculated the molar latent heat of sublimation [18] for zinc acetylacetonate hydrate and anhydrous forms: respectively, $111 \mathrm{~kJ} / \mathrm{mol}$ and $23.5 \mathrm{~kJ} / \mathrm{mol}$.

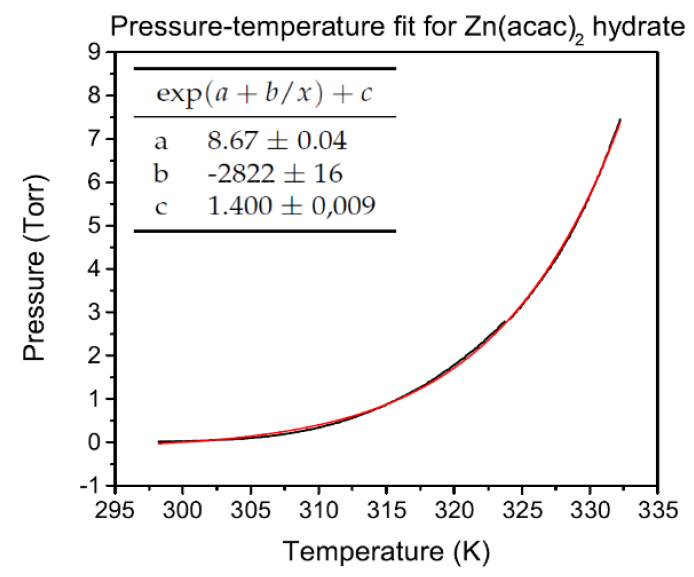

Figure 2. Fit of hydrate zinc acetylacetonate vapor pressure curves.

A thermogravimetric analysis (TGA) of a sample of $14 \mathrm{mg}$ of zinc acetylacetonate was carried out, in conjunction with a measurement of differential scanning calorimetry (DSC). The TGA graph obtained for zinc acetylacetonate hydrate is displayed in Figure 4. 


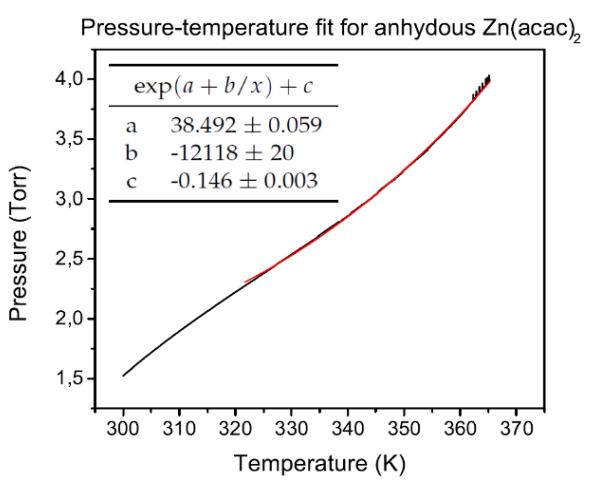

Figure 3. Fit of anhydrous zinc acetylacetonate vapor pressure curves.

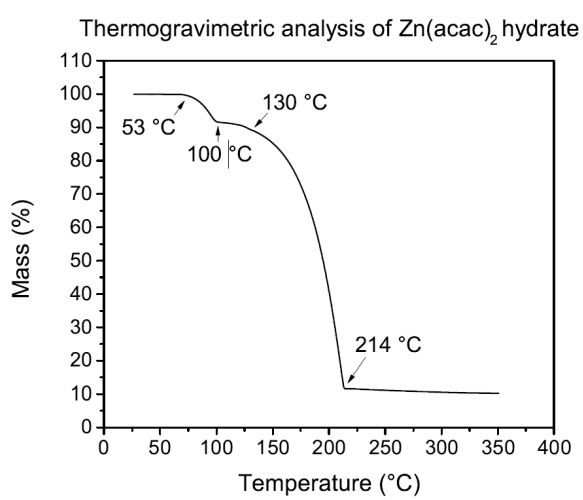

Figure 4. Zinc acetylacetonate TGA in nitrogen atmosphere at atmospheric pressure. Heating rate: $5{ }^{\circ} \mathrm{C} / \mathrm{min}$.

The hydration water (approximately $8 \%$ of the total mass) is lost between $53{ }^{\circ} \mathrm{C}$ and $100{ }^{\circ} \mathrm{C}$. A further decomposition of the compound lasts up to $214{ }^{\circ} \mathrm{C}$, due to parallel reactions including phase transition and the loss via thermal decomposition of acetylacetonate ligands [19]. The DSC analysis was performed to measure the heat flow difference coming from the sample as the temperature increases, making possible the identification of the temperatures at which exothermic or endothermic processes occur for zinc acetylacetonate. Figure 5 shows the heat flow absorbed by the sample while increasing temperature.

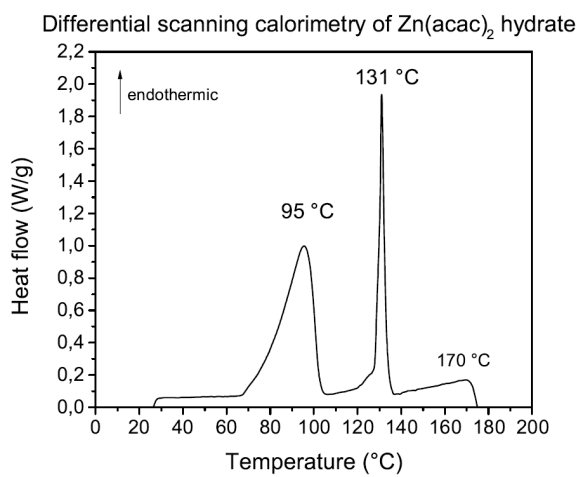

Figure 5. Zinc acetylacetonate DSC in nitrogen atmosphere at atmospheric pressure. Heating rate: $5{ }^{\circ} \mathrm{C} / \mathrm{min}$.

Three main endothermic peaks are visible having different characteristics, indicative of their originating process. In general, broad and asymmetrical peaks, such as those at $95{ }^{\circ} \mathrm{C}$ and $170{ }^{\circ} \mathrm{C}$, are associated with relatively slow processes, such as vapor production. Regarding zinc acetylacetonate hydrate, the peak at $95^{\circ} \mathrm{C}$ may, therefore, be indicative of hydration water loss. The symmetrical 
narrow peak at $131{ }^{\circ} \mathrm{C}$ is associated with the melting of the anhydrous residue, confirmed by studies conducted on anhydrous zinc acetylacetonate [20]; at $170{ }^{\circ} \mathrm{C}$ is located the peak originated by its vaporization. In literature, reheat experiments have been conducted in order to validate peaks nature. It has been shown that the peak at lower temperature was absent during the reheating process, meaning that the peak was due to an irreversible process and could be attributed to water loss. The peak at $131^{\circ} \mathrm{C}$ was also observed by reheating the sample, confirming its reversibility, thus being compatible with melting. The fusion enthalpy per mole of anhydrous zinc acetylacetonate is evaluated by the area under the $131{ }^{\circ} \mathrm{C}$ peak, giving a value of $16.5 \mathrm{~kJ} / \mathrm{mol}$, in good agreement with the literature $(17 \mathrm{~kJ} / \mathrm{mol})$. By adding the substance vaporization enthalpy value calculated from the peak area at $170{ }^{\circ} \mathrm{C}(9.6 \mathrm{~kJ} / \mathrm{mol})$, the sublimation enthalpy of one mole of anhydrous zinc acetylacetonate is $26.1 \mathrm{~kJ}$, in great agreement with the result from vapor pressure curve of anhydrous zinc acetylacetonate ( $23.5 \mathrm{~kJ} / \mathrm{mol}$ ). The molar sublimation enthalpy of anhydrous zinc acetylacetonate is added to the contribution of hydration water loss characteristic peak, centered at $95^{\circ} \mathrm{C}$, in order to obtain the molar enthalpy of the hydrate compound. The calculated value is $114 \mathrm{~kJ}$ per mole of zinc acetylacetonate hydrate, in great agreement with the value measured from vapor pressure curve of $111 \mathrm{~kJ} / \mathrm{mol}$. The previous results allowed to tune the precursor flux from 0.3 to $1 \mathrm{~g} / \mathrm{h}$.

\subsection{Characterization of the Plasma Reactor}

The samples were deposited in different conditions in order to evaluate the films purity and thickness while changing the working parameters. Pressure in the chamber and the composition of the plasma were changed to select the best working conditions to obtain pure films and desired growth rates, by acting on the precursor injection rate. The distance of substrates from the nozzle was maintained constant at $9 \mathrm{~mm}$, in order to overcome the issues of having the substrate too close to the nozzle (with the risk of perturbing the conditions of the plasma chamber) and at the same time to obtain a sufficiently high deposition rate, rarely guaranteed over great distances. At first, optical emission spectroscopy was employed to evaluate the power at which the transition between E-mode and H-mode occurs, in order to determine the range of power suitable for the deposition process. The inductively coupling is more advisable, because there is a better power transfer to plasma, with consequent higher electron density [21]. Therefore, in stable H-mode, the degree of dissociation of the precursor is higher. In Figure 6 are represented the trends of principal optical line intensities as a function of the RF power. Elements of interest are Argon (Ar) and radical oxygen (O), whose selected lines are those at $751 \mathrm{~nm}$ (from $3 \mathrm{~s}^{2} 3 \mathrm{p}^{5}\left({ }^{2} \mathrm{P}_{1 / 2}^{0}\right) 4 \mathrm{~s}$ to $3 \mathrm{~s}^{2} 3 \mathrm{p}^{5}\left({ }^{2} \mathrm{P}_{1 / 2}^{0}\right) 4 \mathrm{p}$ and $763 \mathrm{~nm}$ (from $3 \mathrm{~s}^{2} 3 \mathrm{p}^{5}\left({ }^{2} \mathrm{P}_{3 / 2}^{0}\right) 4 \mathrm{~s}$ to $3 s^{2} 3 p^{5}\left({ }^{2} P_{3 / 2}^{0}\right) 4 p$ ) for Argon and $777 \mathrm{~nm}$ (from $2 s^{2} 2 p^{3}\left({ }^{4} S^{0}\right) 3 s$ to $2 s^{2} 2 p^{3}\left({ }^{4} S^{0}\right) 3 p$ ) and $615 \mathrm{~nm}$ (from $2 s^{2} 2 p^{3}\left({ }^{2} S^{0}\right) 3 p$ to $2 s^{2} 2 p^{3}\left({ }^{2} s^{0}\right) 4 d$ ) for radical oxygen. The intensities were adjusted with the correction factor of the fiber given by the calibration. At a pressure of $8 \mathrm{~Pa}$ in the plasma chamber the transition occurs between $210 \mathrm{~W}$ and $260 \mathrm{~W}$, while, at lower pressure, the $\mathrm{H}$ mode is obtained between $180 \mathrm{~W}$ and $210 \mathrm{~W}$. The transition zone results thicker at higher pressure. In both cases a major increase in density of radical oxygen is observed, less evident with Argon. Analyzing the trends of the same lines augmenting the oxygen in the plasma, the transition occurs rapidly between $200 \mathrm{~W}$ and $210 \mathrm{~W}$, while with lower oxygen quantities it takes place between $90 \mathrm{~W}$ and $115 \mathrm{~W}$. A great increase in radical oxygen density is observed after the transition, less evident when Argon predominates.

As a consequence of this measure, the RF power of $450 \mathrm{~W}$ was chosen for all experiments, in a power range in which plasma is stable in $\mathrm{H}$ mode. During the deposition process, OES analysis was always conducted in order to evaluate precursor flux, by observing the trend of line at $481 \mathrm{~nm}$, associated at $\mathrm{CO}$ molecule, and at $656 \mathrm{~nm}$, associated at $\mathrm{H}$ atom, acting as indexes of precursor dissociation. In Figure 7 is shown the typical trend of $\mathrm{O}, \mathrm{H}$, and $\mathrm{CO}$ lines, while increasing the precursor temperature. By heating it, a major flux of sublimed precursor into the chamber is achieved, and consequently a better dissociation of it in the plasma (visible from the increasing of $\mathrm{CO}$ and $\mathrm{H}$ lines and the corresponding decrease of oxygen intensity). 


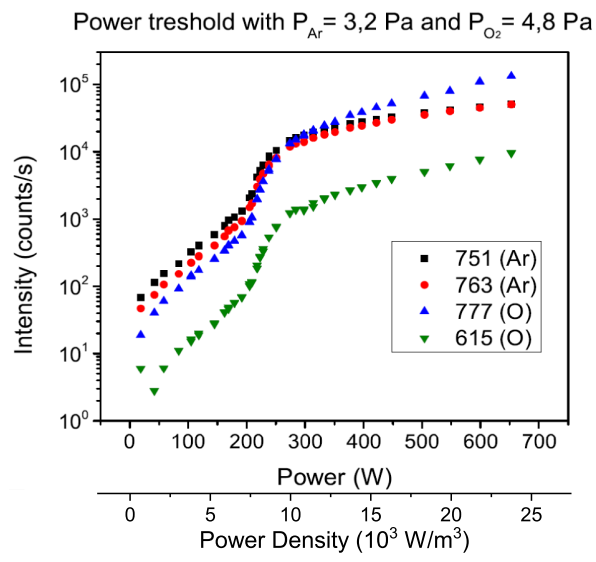

Figure 6. E-H mode transition at $8 \mathrm{~Pa}$, with plasma composition of $40 \% \mathrm{Ar}$ and $60 \% \mathrm{O}_{2}$.

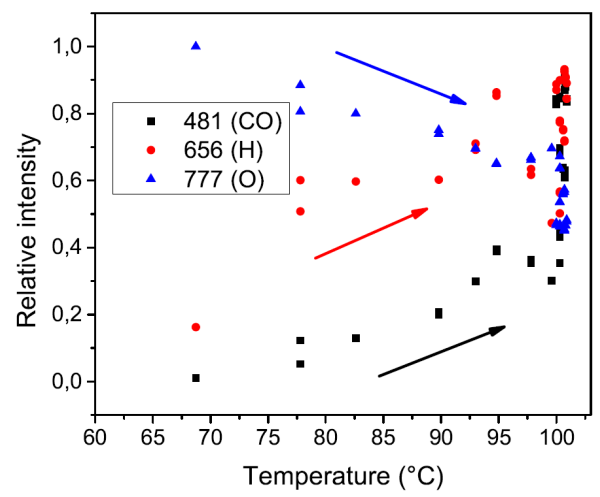

Figure 7. Carbon monoxide, hydrogen and oxygen peaks relative intensity versus precursor temperature.

\subsection{Structural and Chemical Proprieties of the Deposits}

Thin films are deposited on various substrates, mainly silicon and aluminium foil. In particular, the samples grown on aluminium foil are best suited for ATR-FTIR analysis, in order to prevent the dispersive effect of light in a medium, such as silicon [22]. The distance of the substrate from the nozzle was maintained constant at $9 \mathrm{~mm}$.

\subsubsection{Profilometer Analysis}

The sample thickness is measured and averaged in different positions. The deposit distribution is non uniform, a greater amount of material is deposited where the supersonic jet axis intersects the substrate. In order to estimate the profile of the samples, the profiles must be fitted with a formula that takes the angular flow properties into account [23]:

$$
\rho(r, x)=\rho(0, x) \cos ^{2} \theta \cos ^{2}\left(\frac{\pi \theta}{2 \phi}\right)
$$

with $\rho$ particle density, $r$ distance from the point where the measure is taken to the point of incidence of the flow axis, $x$ distance between the nozzle and the substrate, $\phi$ constant equal to 1.5 for the considered gas mixture, and $\theta$ angle given by:

$$
\theta=\arctan \left(\frac{r}{x-x_{0}}\right)
$$

with $x_{0}$ distance between the nozzle and the point from which the flux lines would originate if it was a point source (Figure 8 ). The estimated values of the collected data led to calculation of the maximum thickness of the deposit. 


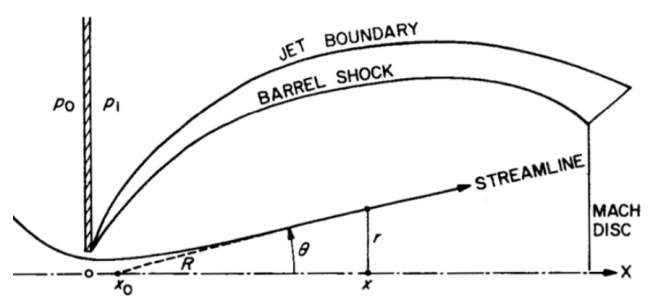

Figure 8. Geometrical parameters of a supersonic jet [23].

In Figure 9, examples of typical deposit profiles with respect to growth rates are displayed. Deposition rates at $9 \mathrm{~mm}$ from the nozzle, with a pressure in the plasma chamber of $8 \mathrm{~Pa}$ and a $40 \%$ Ar and $60 \% \mathrm{O}_{2}$ plasma and a precursor deposition temperature of $100{ }^{\circ} \mathrm{C}$ are of about $30 \mathrm{~nm} / \mathrm{min}$. This suggests that the growth rate can be tuned varying the precursor temperature, in order to obtain the more desired thickness. Only a slightly major precursor flux is obtained by halving the total pressure in the plasma chamber, presumably with consequences on film morphology. The most thick deposit is $475 \mathrm{~nm}$ high with a deposition rate of $34 \mathrm{~nm} / \mathrm{min}$. Defining the aspect ratio as the proportionality between film thickness and its width at half maximum, it can be appreciated that the aspect ratio increases while increasing the deposition rate. Thus, by augmenting the quantity of clusters deposited, the film profile becomes sharper.

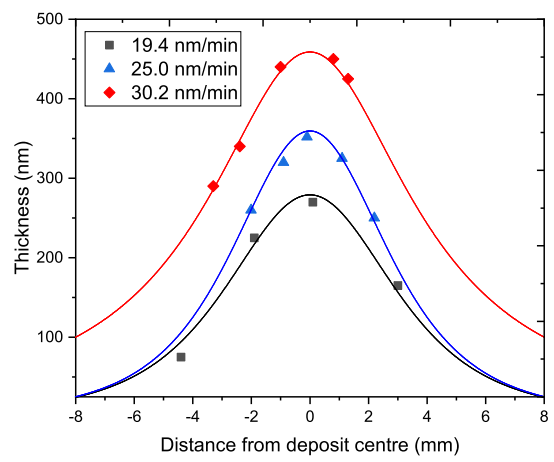

Figure 9. Data and fit of deposits distribution centered at the jet axis as a function of the growth rate.

\subsubsection{ATR-FTIR Analysis}

The vibrational modes of a pure $\mathrm{ZnO}$ crystal at room temperature are $\mathrm{E}_{1}(\mathrm{LO})=589 \mathrm{~cm}^{-1}$, $\mathrm{A}_{1}(\mathrm{LO})=578.5 \mathrm{~cm}^{-1}, \mathrm{E}_{1}(\mathrm{TO})=412 \mathrm{~cm}^{-1}$, and $\mathrm{A}_{1}(\mathrm{TO})=378.5 \mathrm{~cm}^{-1}$. However, considering that the ATR-FTIR spectrometer used in this work is able to investigate only wavenumbers higher than $650 \mathrm{~cm}^{-1}$, a direct measure of $\mathrm{ZnO}$ peaks was not possible, thus only the presence of impurities-bounded zinc could be detected. Therefore, the ATR-FTIR analysis was conducted in order to assess the purity of the films and identify the presence of any carbonaceous groups, residues of an incomplete oxidation of the precursor.

Figure 10 displays the ATR-FTIR spectra collected on samples deposited starting from different plasma mixture at the same total treatment pressure. The broad peak at $3200 \mathrm{~cm}^{-1}$ is due to the stretching of the $\mathrm{OH}$ bond, the last one indicative of water presence in the mixture. The stronger the hydrogen bonds between the molecules, the more this band will be shifted to lower wavenumbers, since these bonds tend to weaken the $\mathrm{OH}$ bond, facilitating its stretching [24]. A peak in the lower range at $1524-1691 \mathrm{~cm}^{-1}$ is due to $\mathrm{OH}$ bending. Several components are related to carbonaceous functional groups. At $2339 \mathrm{~cm}^{-1}$, a peak that is assigned to $\mathrm{CO}_{2}$ suggests that carbon dioxide molecules are formed by reactions involving the organic precursor oxidation and adsorbed on the film surface [25]. Vibrational modes related to the zinc carbonate functional groups generate absorption peaks at $1077 \mathrm{~cm}^{-1}$ and $831 \mathrm{~cm}^{-1}$ due to the $\mathrm{CO}_{3}$ stretching and a peak centred at $712 \mathrm{~cm}^{-1}$ due to the $\mathrm{CO}_{3}$ bending [26]. When comparing the spectra, one can surmise that oxygen is fundamental to assure 
an efficient precursor fragmentation, indeed the higher Ar pressure is the more $-\mathrm{CH}_{x}$ groups are retained in films [27]. Moreover, at low oxygen concentration, $\mathrm{CO}$ absorption bands are not present. Therefore augmenting the quantity of oxygen in the plasma, a better purity is achieved, and it is almost total when oxygen constitutes the $75 \%$ of the plasma. Finally, it could be observed a strong broad band absorption in the spectrum of the most pure sample. This is in good agreement with a hypothesis of polycrystalline films, in which the light absorption is dominated by electron-scattering mechanisms that are linked to grain boundaries in the materials and directly proportional to a power of the incident light wavelength.

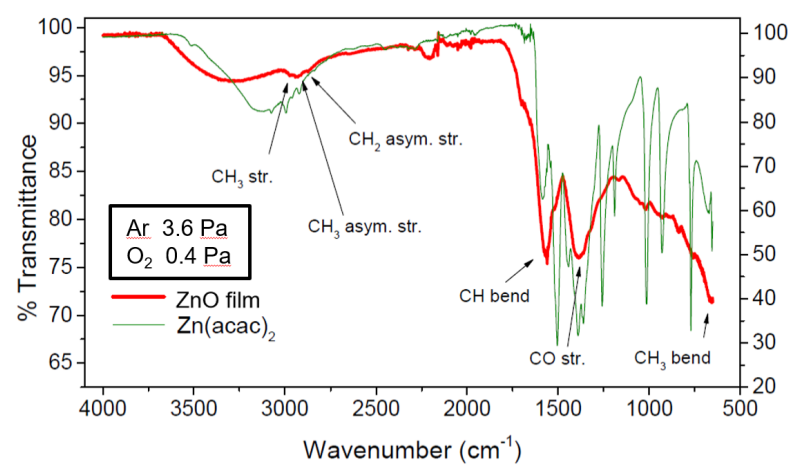

(a) Attenuated total reflection Fourier transform infrared spectroscopy (ATR-FTIR) spectrum of a 3.6 $\mathrm{Pa} \mathrm{Ar}+0.4 \mathrm{~Pa} \mathrm{O}_{2}$ sample.

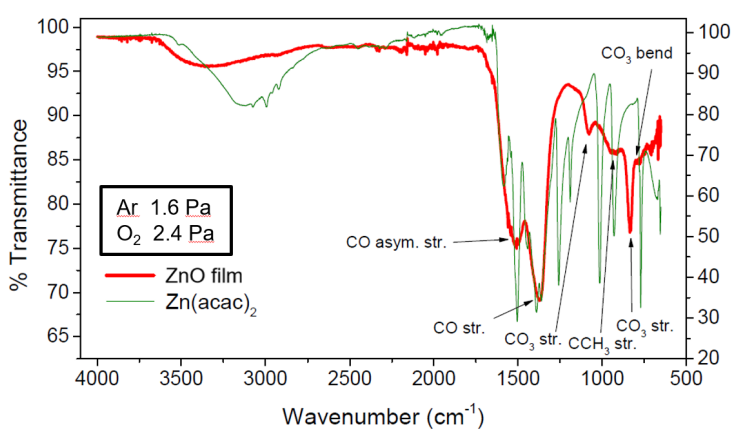

(b) ATR-FTIR spectrum of a 1.6 $\mathrm{Pa} \mathrm{Ar}+2.4 \mathrm{~Pa} \mathrm{O}_{2}$ sample.

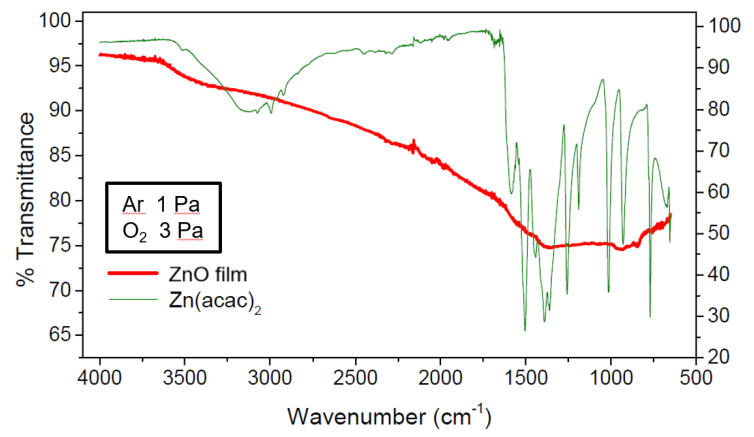

(c) ATR-FTIR spectrum of a $1 \mathrm{~Pa} \mathrm{Ar}+3 \mathrm{~Pa} \mathrm{O}_{2}$ sample.

Figure 10. ATR-FTIR analysis of samples deposited (in red) with different plasma compositions at $4 \mathrm{~Pa}$ total treatment pressure with respect to the precursor spectrum (in green).

\subsubsection{The Annealing Process}

Annealing alters the physical and chemical properties of a material. Atoms diffuse within the solid material thanks to the thermal energy, so that the material progresses towards its equilibrium state. Simultaneously, the volatile impurities are removed. The annealing process has been conducted in an oven at atmospheric pressure by heating samples for an hour at $500^{\circ} \mathrm{C}$ for films deposited on silicon, at $280^{\circ} \mathrm{C}$ for films deposited on aluminium foil. The lower temperature was used in order to prevent any damage to the aluminum foil and because the temperature was sufficient for an effective removal of impurities. ATR-FTIR analyses confirm the removal of a large part of impurities after the annealing treatments.

\subsubsection{Structural and Morphological of the Annealed Films}

For zinc oxide, the zone-centre optical phonons can be classified according to the following irreducible representation: $\Gamma_{o p t}=\mathrm{A}_{1}+\mathrm{E}_{1}+2 \mathrm{E}_{2}+2 \mathrm{~B}_{1}$. In this non-centrosymmetric material, $\mathrm{IR}$ and 
Raman activities are not mutually exclusive properties. The polar modes $\mathrm{A}_{1}$ and $\mathrm{E}_{1}$ are IR as well as Raman active. Furthermore, Raman activity applies for the non-polar modes $\mathrm{E}_{1}, \mathrm{E}_{2}^{\text {high }}, \mathrm{E}_{2}^{\text {low }}$. The $\mathrm{B}_{1}$ modes are neither Raman nor IR active. As derived from the Raman tensors, all of the Raman active modes can be observed in backscattering geometry, except for the $\mathrm{E}_{1}(\mathrm{LO})$ mode. Table 2 shows the frequency and the symmetry of the fundamental optical modes in wurtzite $\mathrm{ZnO}[28,29]$. Figure 11 shows the Raman spectrum of an annealed thin film in a $\mathrm{Ar} 1.6 \mathrm{~Pa}+\mathrm{O}_{2} 2.4 \mathrm{~Pa}$ mixture. The measured absorption is in excellent agreement with peaks enlisted in Table 2. Therefore, the nature of the deposited films can be reasonably attributed to zinc oxide in its wurtzite structure.

Table 2. Frequency and simmetry of the fundamental optical modes in wurtzite $\mathrm{ZnO}$.

\begin{tabular}{lc}
\hline Wavenumber $\left(\mathrm{cm}^{-\mathbf{1}}\right)$ & Simmetry \\
\hline 101 & $\mathrm{E}_{2}^{\text {low }}$ \\
208 & $2 \mathrm{TA}, \mathrm{E}_{2}^{\text {low }}$ \\
332 & $\mathrm{E}_{2}^{\text {high }}, \mathrm{E}_{2}^{\text {low }}$ \\
308 & $\mathrm{~A}_{1}(\mathrm{TO})$ \\
408 & $\mathrm{E}_{1}(\mathrm{TO})$ \\
437 & $\mathrm{E}_{2}^{\text {high }}$ \\
574 & $\mathrm{~A}_{1}(\mathrm{LO})$ \\
584 & $\mathrm{E}_{1}(\mathrm{LO})$ \\
\hline
\end{tabular}

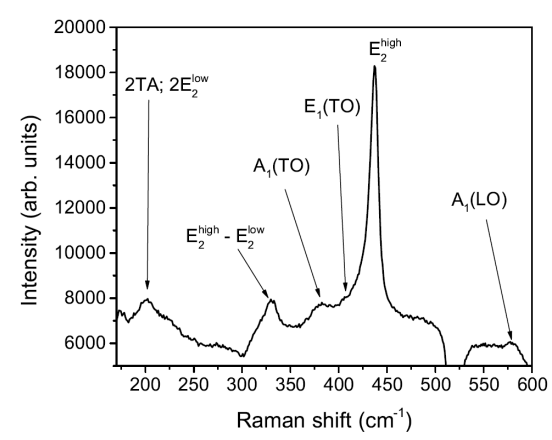

Figure 11. Raman spectrum of the thickest film deposited, to overwhelm the silicon substrate noise.

AFM and SEM analyses are performed to investigate the film surface morphology. Figure 12 shows the results of an AFM analysis performed on a thin film deposited at $\mathrm{P}_{\mathrm{p}}=8 \mathrm{~Pa}$. The morphological structure of the film is displayed in a planar and 3D view, grains are visible on sample surface. The surface roughness is calculated as the root mean square of the height distribution [30] and reported in Table 3. The maximum height is $22 \mathrm{~nm}$ over the surface.

Figure 13 shows SEM images performed on annealed samples. The samples are deposited in two different total treatment pressure in the plasma chamber (the first $\mathrm{P}_{\mathrm{p}}=8 \mathrm{~Pa}$, the second $\mathrm{P}_{\mathrm{p}}=4 \mathrm{~Pa}$ ) keeping fixed the $\mathrm{Ar} / \mathrm{O}_{2}$ ratio in the mixture. In both cases, films show a uniform distribution of columnar structures with impervious grain boundaries. The obliqueness of the structures is due to shadowing effect related to the geometry of the supersonic jet. Columnar structures are well defined, since their first deposition steps and increase their dimensions as the film grows. Structures are larger for sample deposited with a lower pressure in the plasma chamber. This is in agreement with the major precursor flux at lower pressures behaviour, as previously discussed. For films deposited at $8 \mathrm{~Pa}$, the columns show a diameter that ranges from about $15 \mathrm{~nm}$ to $25 \mathrm{~nm}$ while growing in height. For samples at lower pressure, the diameters range from about $18 \mathrm{~nm}$ to $40 \mathrm{~nm}$. The polycrystalline columns and the porosity of the films could be of great interest in application such as sensor systems, for the large surface area to volume ratio, and solar cells, since a favourite current flow direction enhances power conversion efficiency [31]. 


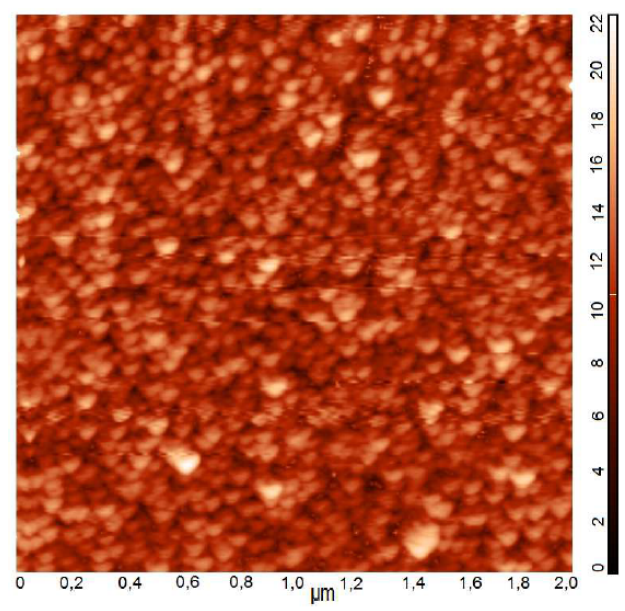

(a) Planar view, 2D surface plot.

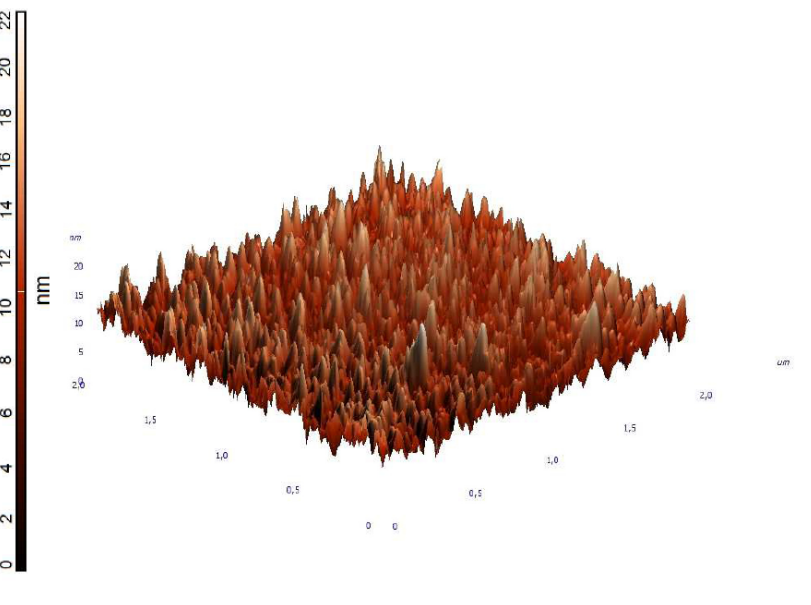

(b) 3D surface plot.

Figure 12. Example of Atomic Force Microscopy (AFM) results of a deposited film.

Table 3. Surface roughness (RMS) and grains size calculated from the AFM analysis.

\begin{tabular}{ccc}
\hline Pressure $\left.\mathbf{A r} / \mathbf{O}_{2} \mathbf{( P a}\right)$ & RMS $(\mathbf{n m})$ & Grain Size $(\mathbf{n m})$ \\
\hline $3.2 / 4.8$ & $4.1 \pm 0.4$ & $30-40$ \\
$1.6 / 2.4$ & $4.5 \pm 0.4$ & $30-40$ \\
$1 / 3$ & $6.2 \pm 0.5$ & $30-40$ \\
\hline
\end{tabular}

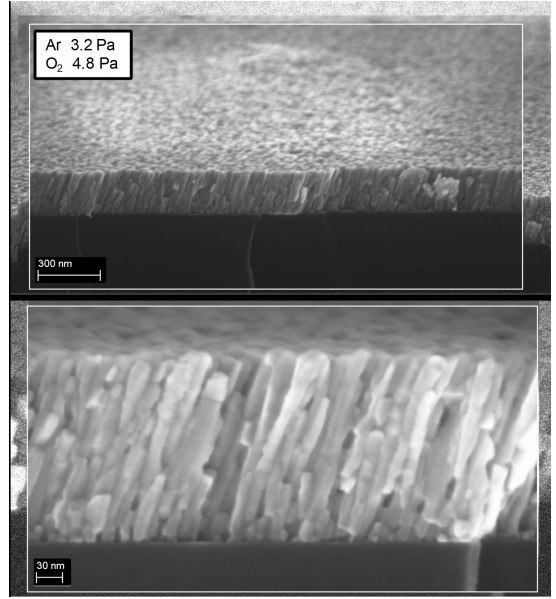

(a) Thin film deposited from a $3.2 \mathrm{~Pa} \mathrm{Ar}+4.8 \mathrm{~Pa} \mathrm{O}$ starting mixture.

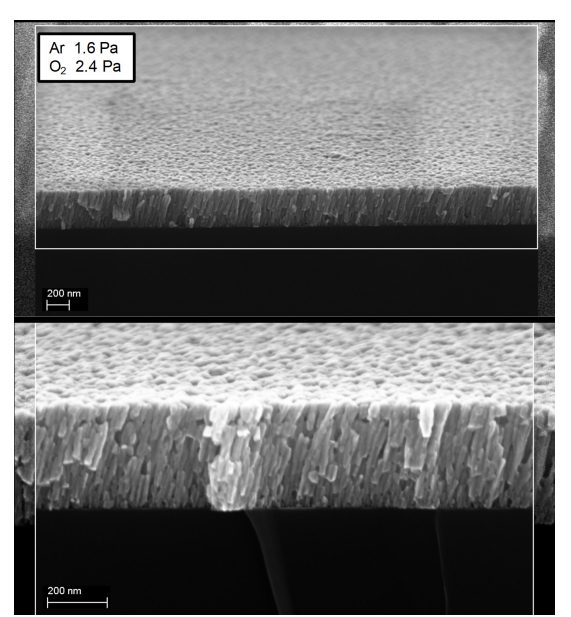

(b) Thin film deposited from a $1.6 \mathrm{~Pa} \mathrm{Ar}+2.4 \mathrm{~Pa} \mathrm{O}_{2}$ starting mixture.

Figure 13. SEM micrographs of the columnar structures in two samples different in the starting plasma pressure of $8 \mathrm{~Pa}$ in panel (a) and $4 \mathrm{~Pa}$ in panel (b).

\section{Summary and Conclusions}

In this paper, an innovative deposition technique for the controlled deposition of zinc oxide nanostructured thin films was presented. The novelty of the proposed approach is the segmentation of the growth process in two separate steps: the precursor dissociation and metal oxide particles formation in a reactive cold plasma environment; the film nucleation and assembling controlled by means of a supersonic seeded jet impinging on a substrate. Zinc acetylacetonate was chosen as a metalorganic zinc precursor, despite its wide use very poor literature found on its proprieties. A thermodynamic experimental characterization of $\mathrm{Zn}(\mathrm{acac})_{2}$ was first performed and the best oxygen 
concentration to efficiently produce $\mathrm{ZnO}$ was estimated. Having knowledge of vapor pressure curves, it was possible to relate the heating temperature to the pressure of the precursor, thus permitting the precursor injection with a controllable flux.

OES revealed the transition between the capacitively coupled mode (E mode) and the inductively coupled mode (H mode), the latter suitable for depositions, being characterized by a major quantity of electrons and reactive species. OES results indicate that the optimal total pressure in the first chamber is in the 4-8 Pa range and the pressure in the second chamber between 0.1-0.4 Pa, in order to obtain a supersonic expansion length large enough to allow depositions at distances of 3-25 $\mathrm{mm}$ from the nozzle, along the jet axis. Because of the nature of the expansion, a decrease in the deposition rate was expected by moving away from the nozzle. Therefore, the distance of the substrate from the nozzle was set at $9 \mathrm{~mm}$. The total pressure and gas composition variation influences film morphology and chemical structure. The films characteristics were evaluated by means of contact profilometry, ATR-FTIR spectroscopy, Raman spectroscopy, AFM and SEM. The thickness of films were less than $500 \mathrm{~nm}$ and the maximun growth rate was $34 \mathrm{~nm} / \mathrm{min}$. It was demonstrated that an increase in aspect ratio is observed increasing the deposition rate. ATR-FTIR was employed in order to obtain information about the film purity. Many $\mathrm{CH}_{x}$ and $\mathrm{CO}_{x}$ impurities were observed in several samples, a complete combustion occurs only at high oxygen quantities. ATR-FTIR spectra after the thermal treatment showed the excellent removal of any impurity. The Raman spectroscopy confirmed that the zinc oxide is coordinated in a wurtzite structure, the most common and stable form for applications. The wurtzite structure is non-centrosymmetric, so crystals have piezoelectricity properties. This is a basic prerequisite for applications, such as Surface Acoustic Wave (SAW) devices and actuators. AFM measurements revealed the presence of grains with radii of the order of tens nanometers on the film surface, having roughness of the order of nanometers. Thin films morphology is organized in loose packed columns, as assessed by the SEM micrographs, with largest structures obtained at lower pressures in the plasma chamber. Because of these regular and columnar structures, deposited films are promising for a future study on their applicability as sensors, both gas sensors and biosensors, photocatalysts for contaminants degradation, purification and solar cells. Further investigations will be directed to the conductivity, gas sensitivity and selectivity, piezoresponse, and transparency of the nanostructured $\mathrm{ZnO}$ thin films.

Author Contributions: Conceptualization, C.C. and C.R.; methodology, E.D.; validation, C.C., C.R. and E.D.; formal analysis, C.C., C.R. and V.M.; investigation, C.C., C.R. and V.M.; resources, C.R.; data curation, E.D.; writing-original draft preparation, C.C.; writing-review and editing, C.C. and C.R.; visualization, E.D.; supervision, C.C. and C.R.; project administration, C.R.; funding acquisition, C.R. All authors have read and agreed to the published version of the manuscript.

Funding: This research was partially funded by CARIPLO Fundation ("Produzione di film sottili e nanostrutture gerarchiche di ossidi, semiconduttori e metalli mediante un plasma supersonico reattivo" Project, 2010-2013, No. 2010.0623).

Acknowledgments: We gratefully acknowledge the technical support of Alessandro Mietner in the device development and experiment execution.

Conflicts of Interest: The authors declare no conflict of interest.

\section{References}

1. Zanini, S.; Grimoldi, E.; Riccardi, C. Development of controlled releasing surfaces by plasma deposited multilayers. Mater. Chem. Phys. 2013, 138, 850-855. [CrossRef]

2. Zanini, S.; Polissi, A.; Maccagni, E.A.; Dell'Orto, E.C.; Liberatore, C.; Riccardi, C. Development of antibacterial quaternary ammonium silane coatings on polyurethane catheters. J. Colloid Interface Sci. 2015, 451, 78-84. [CrossRef] [PubMed]

3. Guozhong, C. Nanostructures and Nanomaterials: Synthesis, Properties and Applications; World Scientific: Singapore, 2004.

4. Klingshirn, C.F.; Waag, A.; Hoffmann, A.; Geurts, J. Zinc Oxide: From Fundamental Properties towards Novel Applications; Springer Science \& Business Media: Berlin, Germany, 2010; Volume 120. 
5. Özgür, Ü.; Alivov, Y.I.; Liu, C.; Teke, A.; Reshchikov, M.A.; Doğan, S.; Avrutin, V.; Cho, S.J.; Morkoç, H. A comprehensive review of $\mathrm{ZnO}$ materials and devices. J. Appl. Phys. 2005, 98, 041301. [CrossRef]

6. Lu, H.; Wang, S.; Zhao, L.; Li, J.; Dong, B.; Xu, Z. Hierarchical ZnO microarchitectures assembled by ultrathin nanosheets: Hydrothermal synthesis and enhanced photocatalytic activity. J. Mater. Chem. 2011, 21, 4228-4234. [CrossRef]

7. Zhang, H.; Sun, J.; Dagle, V.L.; Halevi, B.; Datye, A.K.; Wang, Y. Influence of ZnO facets on Pd/ZnO catalysts for methanol steam reforming. ACS Catal. 2014, 4, 2379-2386. [CrossRef]

8. Gao, G.; Yu, L.; Vinu, A.; Shapter, J.G.; Batmunkh, M.; Shearer, C.J.; Yin, T.; Huang, P.; Cui, D. Synthesis of ultra-long hierarchical $\mathrm{ZnO}$ whiskers in a hydrothermal system for dye-sensitised solar cells. RSC Adv. 2016, 6, 109406-109413. [CrossRef]

9. Ramadan, R.; Torres-Costa, V.; Martín-Palma, R.J. Fabrication of Zinc Oxide and Nanostructured Porous Silicon Composite Micropatterns on Silicon. Coatings 2020, 10, 529. [CrossRef]

10. Dell'Orto, E.; Caldirola, S.; Sassella, A.; Morandi, V.; Riccardi, C. Growth and properties of nanostructured titanium dioxide deposited by supersonic plasma jet deposition. Appl. Surf. Sci. 2017, 425, 407-415. [CrossRef]

11. Caldirola, S.; Roman, H.; Riccardi, C. Ion dynamics in a supersonic jet: Experiments and simulations. Phys. Rev. E 2016, 93, 033202. [CrossRef]

12. Biganzoli, I.; Fumagalli, F.; Di Fonzo, F.; Barni, R.; Riccardi, C. A supersonic plasma jet source for controlled and efficient thin film deposition. J. Mod. Phys. 2012, 3, 1626. [CrossRef]

13. Friedrich, J. The Plasma Chemistry of Polymer Surfaces: Advanced Techniques for Surface Design; John Wiley \& Sons: Hoboken, NJ, USA, 2012.

14. Baxter, J.B.; Aydil, E.S. Metallorganic chemical vapor deposition of $\mathrm{ZnO}$ nanowires from zinc acetylacetonate and oxygen. J. Electrochem. Soc. 2008, 156, H52. [CrossRef]

15. Vovna, V.; Korochentsev, V.; Dotsenko, A. Electronic structures and photoelectron spectra of zinc (II) bis- $\beta$-diketonates. Russ. J. Coord. Chem. 2012, 38, 36-43. [CrossRef]

16. Wolden, C.A. The role of oxygen dissociation in plasma enhanced chemical vapor deposition of zinc oxide from oxygen and diethyl zinc. Plasma Chem. Plasma Process. 2005, 25, 169-192. [CrossRef]

17. Macknick, A.; Prausnitz, J. Vapor pressures of heavy liquid hydrocarbons by a group-contribution method. Ind. Eng. Chem. Fundam. 1979, 18, 348-351. [CrossRef]

18. Lucchesi, C.A.; Lewis, W. Latent heat of sublimation of terephthalic acid from differential thermal analysis data. J. Chem. Eng. Data 1968, 13, 389-391. [CrossRef]

19. Musić, S.; Šarić, A.; Popović, S. Formation of nanosize ZnO particles by thermal decomposition of zinc acetylacetonate monohydrate. Ceram. Int. 2010, 36, 1117-1123. [CrossRef]

20. Coates, P.D. A Study of the Preparation and Pyrolysis of $\beta$-diketonate and Carboxylate Precursors to Semiconducting Zinc Oxide Films. Ph.D. Thesis, Durham University, Durham, UK, 1994.

21. Barni, R.; Zanini, S.; Riccardi, C. Diagnostics of reactive RF plasmas. Vacuum 2007, 82, 217-219. [CrossRef]

22. Zanini, S.; Riccardi, C.; Orlandi, M.; Grimoldi, E. Characterisation of $\mathrm{SiO}_{x} \mathrm{C}_{y} \mathrm{H}_{z}$ thin films deposited by low-temperature PECVD. Vacuum 2007, 82, 290-293. [CrossRef]

23. Ashkenas, H.; Sherman, F.S. Structure and Utilization of Supersonic Free Jets in Low Density Wind Tunnels; NTRS—NASA Technical Reports Server: Washington, DC, USA, 1965.

24. Zanini, S.; Massini, P.; Mietta, M.; Grimoldi, E.; Riccardi, C. Plasma treatments of PET meshes for fuel-water separation applications. J. Colloid Interface Sci. 2008, 322, 566-571. [CrossRef]

25. Hlaing Oo, W.; McCluskey, M.; Lalonde, A.; Norton, M. Infrared spectroscopy of ZnO nanoparticles containing $\mathrm{CO}_{2}$ impurities. Appl. Phys. Lett. 2005, 86, 073111. [CrossRef]

26. Pholnak, C.; Sirisathitkul, C.; Suwanboon, S.; Harding, D.J. Effects of precursor concentration and reaction time on sonochemically synthesized ZnO nanoparticles. Mater. Res. 2014, 17, 405-411. [CrossRef]

27. Zanini, S.; Ziano, R.; Riccardi, C. Stable Poly (Acrylic Acid) films from acrylic acid/argon plasmas: Influence of the mixture composition and the reactor geometry on the thin films chemical structures. Plasma Chem. Plasma Process. 2009, 29, 535. [CrossRef]

28. Damen, T.C.; Porto, S.; Tell, B. Raman effect in zinc oxide. Phys. Rev. 1966, 142, 570. [CrossRef]

29. Cuscó, R.; Alarcón-Lladó, E.; Ibanez, J.; Artús, L.; Jiménez, J.; Wang, B.; Callahan, M.J. Temperature dependence of Raman scattering in ZnO. Phys. Rev. B 2007, 75, 165202. [CrossRef] 
30. Grimoldi, E.; Zanini, S.; Siliprandi, R.; Riccardi, C. AFM and contact angle investigation of growth and structure of pp-HMDSO thin films. Eur. Phys. J. D 2009, 54, 165-172. [CrossRef]

31. Trifiletti, V.; Ruffo, R.; Turrini, C.; Tassetti, D.; Brescia, R.; Di Fonzo, F.; Riccardi, C.; Abbotto, A. Dye-sensitized solar cells containing plasma jet deposited hierarchically nanostructured $\mathrm{TiO}_{2}$ thin photoanodes. J. Mater. Chem. A 2013, 1, 11665-11673. [CrossRef] 